\title{
Thoracic Spine Spasms Secondary to Hemorrhagic Intestinal Ulcer
}

\author{
Curtis W. Slipman, MD, William S. Whyte II, MD, Gary R. Lichtenstein, MD, Dave Lenrow, MD, \\ Debra Braverman, MD, Mark Ellen, MD, and Edward J. Vresilovic, MD, PhD
}

\begin{abstract}
A case of thoracic spine spasms secondary to a bleeding duodenal ulcer is presented. A 41-year-old male with 14week history of thoracic spine spasm was treated with bed rest, spinal manipulation, physical therapy, medication, and a thoracolumbar brace. Subsequently, a provocative thoracic discogram performed at T9-T10 created periscapular pain and also reproduced the presenting thoracic spasms. Intradiscal electrothermal annuloplasty (IDET) was performed at the T9-T10 level, but without sustained relief. The patient presented to a spine center for evaluation. The diagnosis of thoracic discogenic disease was suspected. A second provocative thoracic discogram was performed and failed to reproduce his thoracic spasms. Three weeks after
\end{abstract}

being referred to a chronic pain management physician, the patient presented to a local emergency room with hematemesis. An endoscopic evaluation revealed a bleeding duodenal ulcer. Following medical treatment of the duodenal ulcer with a proton pump inhibitor the patient had complete resolution of his thoracic spasms. This represents the first reported case of thoracic spine spasms as an initial presenting symptom of a bleeding peptic ulcer.

Key Words: Thoracic spine muscle spasms, thoracic spine pain, intestinal ulcers, peptic ulcer disease, hemorrhagic ulcers
Muscular spasms are an involuntary local contraction that involves an entire muscle, which are not relieved with voluntary effort $(1,2)$. Spasms typically develop as a reflex reaction to an irritative focus (3). Internal disc disruption, facet joint syndrome, radiculopathy, or osseous pathology are some of the disorders that can elicit spasms in the thoracic region. Typically, these etiologies present with concurrent pain when spasms are present. An uncommon etiology of thoracic pain and spasm is pathology intrinsic to the gastrointestinal system such as duodenal ulcer, gastric ulcer, pancreatitis, ulcerative colitis, and cholecystitis (4-6). Peptic ulcers are holes extending through the mucosa into the muscularis propria of the esophagus, stomach, or duodenum. Thoracic spinal pain may be the only initial symptom of hemorrhagic duodenal ulcer (7).To date, thoracic spine spasm without back pain has not been reported as an initial presenting symptom for peptic ulcer disease.

From the departments of Rehabilitation Medicine, Orthapaedics and Gastroenterology, University of Pennsylvania Health System, Philadelphia, Pennsylvania. Address correspondence: Curtis W. Slipman, MD, Director, The Penn Spine Center, Ground Floor White Bldg., 3400 Spruce Street, Philadelphia, PA 19104 Email: slipman@mail.med.upenn.edu
We report a case of a patient presenting with spasms of the thoracic muscles without concurrent pain due to hemorrhagic peptic ulcer disease.

\section{CASE REPORT}

A 41-year-old man presented to us approximately five months following the spontaneous onset of acute thoracic spasms. These spasms were localized to the $8^{\text {th }}$ and $9^{\text {th }}$ thoracic vertebral level and extended approximately two centimeters caudal to the inferior angle of the right scapula. He described the spasms as a "severe cramp", but denied a concurrent burning, aching, or throbbing quality. Two days after their onset the intensity of the spasms dramatically increased. He was assessed at a local emergency room. Ibuprofen $600 \mathrm{mg}$, cyclobenzaprine, and several days of bed rest were prescribed. Two weeks later, there was no improvement, leading to a chiropractic evaluation. A series of manipulations were performed over a four week period. He simultaneously participated in physical therapy for a three month duration. This program provided no relief of the patients' symptomatology.

Two months after the onset of the spasms, the patient visited a chronic pain management clinic. Following his initial evaluation the patient was simultaneously given 
numerous prescriptions including: an additional three weeks of physical therapy, controlled-release morphine sulfate, oxycodone with acetaminophen, rofecoxib, gabapentin, and a thoracolumbar corset brace to be worn during unprotective activities such as driving. Prior to starting physical therapy, the patient underwent three trigger point injections into the parathoracic musculature. This intervention provided no immediate or subsequent relief of his spasms. Activities in therapy were oriented toward improving the condition of his thoracic musculature with dynamic stabilizing exercises. It was explained to the patient, by his therapist, that his spasms were the result of deconditioning and inflexibility. After two weeks the patient discontinued therapy because the back spasms were not improving. When the patient returned for his follow-up visit his treating physician explained that the back spasms were due to a "torn disc versus a pinched nerve". A therapeutic right T9 nerve root block was attempted, but was unsuccessful. The patient then underwent provocative discography at the T8-9, T9-10, and T1011 levels. Stimulation of the intervertebral disc at T9-10 reproduced the patient's spasms and created stabbing-like pain just inferior to the right scapula. A post-discography computerized axial tomography (CAT) scan demonstrated bilateral posterolateral tears of the annulus at T910, a right posterolateral annular tear at T8-9, and a left posterolateral annular tear at T10-11. Four weeks later, the patient underwent intradiscal electrothermal annuloplasty (IDET) at the T9-10 level. This provided 90 percent relief of his spasms for the first six weeks following the IDET procedure. It did not relieve any of the patients' new periscapular pain that was created by and continued after discography. After this six week interval the patient returned to work. One week later the spasms returned to approximately 50 percent of their pre-IDET intensity level. It was at this time the patient began experiencing pain in this same region for the first time. The patient described the pain as having a constant stabbing quality. Activities that exacerbated the thoracic pain were sitting or driving greater than 15 minutes. The pain was partially relieved by resting in the lateral decubitus position.

Five months later the patient was referred to our interdisciplinary Spine Center. His review of symptoms revealed no history of abdominal pain, hematemesis, heartburn, or melena. The patient also denied the use of non-steroidal anti-inflammatory drugs prior to his initial visit to the emergency room. Physical examination revealed a well nourished male in no apparent distress. He was 5' 8', tall, 150 pounds, and had a blood pressure of $110 /$
$80 \mathrm{mmHg}$. Lower extremity muscle girth appeared symmetric proximally and distally. Dorsalis pedis and posterior tibial pulses were graded $2+$ and symmetric. There was no tenderness to palpation over the thoracic spinous processes or musculature. Lumbar spine flexion was not painful and was achieved with a long finger to floor distance of five $\mathrm{cm}$. Lumbar extension, left and right side bending was not pain or spasm provoking. Pelvic rock and sustained hip flexion was negative. Sacroiliac joint provocative maneuvers did not induce pain. Deep tendon reflexes were graded $2+/ 4$ and symmetric throughout the upper and lower limbs. Sensation was intact to pinprick, light touch and proprioception in all the limbs. Sharp sensation and light touch was preserved in all thoracic dermatomes. No myotomal deficits were present, and the patient was noted to heel and toe walk without difficulty. Plantar reflexes were downgoing, Hoffman's was negative, and clonus was absent in the bilateral lower extremities. Plain radiographs of the thoracic spine demonstrated a congenital fusion of the posterior aspect of the right $10^{\text {th }}$ and $11^{\text {th }}$ ribs. There was a very mild depression of the superior endplate of vertebral bodies T10, T11, and T12. Magnetic resonance imaging of the cervical and thoracic spine showed a mild central disc herniation at C3-4, small left lateral disc herniations at T5-6,T6-7, and T7-8. At T9-10, a small right-sided disc herniation was present minimally compressing the spinal cord. There was no associated nerve root compression. No significant foraminal or central canal stenosis was seen. No evidence of discitis, abcess, or cyst formation was present.

The old discography, post-discograpy CAT scan, and IDET fluoroscopic films were subsequently obtained. The anterior-posterior and lateral discography films demonstrated good placement of all needles. The CAT scan demonstrated a right posterolateral tear at T8-9, a bilateral posterolateral tear at T9-T10, and a left posterolateral tear at T10-11. Review of the IDET fluoroscopic films showed proper placement of the thermal catheter.

After assimilating the information obtained from prior records, his history, and our physical exam the diagnosis of thoracic discogenic disease was suspected. The patient's symptoms were considered to be originating from the same thoracic disc level (T9-10) or emanating from the level above (T8-9). The patient underwent a repeat provocative thoracic discogram to evaluate T8-9 and T9-10. This failed to reproduce his usual symptoms. Due to our inability to provide a specific diagnosis, the patient was referred for pain modulation techniques. The patient received intensive training on how to cope with pain, in- 


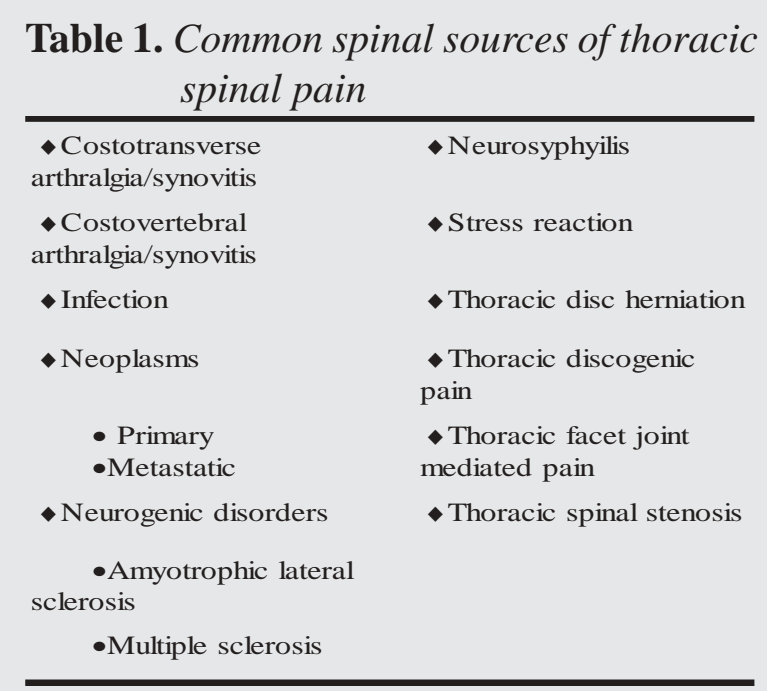

cluding distraction and relaxation techniques. All previous opioid medications were discontinued and dilaudid rectal suppositories were dispensed.

Approximately three weeks later, the patient suffered an episode of hematemesis, and worsening of the thoracic spine spasm and the post-discography scapular pain. He was evaluated at a local emergency room. Workup revealed a hemorrhagic duodenal ulcer and a gastroenterologist was consulted. Four weeks later, following treatment of the duodenal ulcer with omeprazole $20 \mathrm{mg} /$ day, the patient experienced complete resolution of thoracic back spasms and pain.

\section{DISCUSSION}

An enormous amount of literature exists about thoracic spine pain, however, there is a paucity of literature about isolated thoracic spasms (7). Although it is well understood that thoracic spinal pain can be either spinal or extraspinal in origin (Tables 1 and 2), a similar comment cannot be offered concerning thoracic spasms. Perhaps the reason there is no literature about isolated thoracic spasms is, because to date, it has not been recognized as a potential symptom of intra-abdominal pathology or its occurrence is rare.

Pain experienced in a part of the body that is considerably removed from the tissue causing the pain is termed referred pain. Any stimulus that excites pain nerve endings in diffuse areas of the viscera causes visceral pain. Such stimuli include ischemia of the visceral tissue, chemical damage to the surfaces of the viscera, spasm of the smooth intestinal muscle, distention of the intestine, or stretching of the intestinal ligaments (8). Essentially all true visceral pain originating in the abdominal cavities is transmitted through small unmyelinated sensory nerve fibers that run with the sympathetic nerves; type $C$ nerve fibers. These fibers travel from the duodenum through the superior mesenteric ganglion and celiac ganglion. From these ganglions, the pain fibers travel to the T8 thru T12 segments via the lesser or lowest thoracic splanchnic nerves (9). The mechanism of muscle spasm has not been elucidated to complete satisfaction even in experimental animals, but perhaps it is through this aforementioned neural pathway that spasms were elicited in the parathoracic musculature.

Table 2. Common extraspinal sources of thoracic spinal pain

\begin{tabular}{llll}
\hline Intrathoracic & Intra-abdominal & Retroperitoneal & Miscellaneous \\
\hline Angina pectoris & Abscess & Aneurysm & Herpes zoster \\
Aortic aneurysm & Biliary colic & Pyelonephritis & Polymalgia rheumatica \\
Carcinoma & Cholecystitis & Tumor & Rib fractures \\
Mitral valve prolapse & Hepatitis & Ureteral colic & \\
Myocardial infarct & Hernia & & \\
Pericarditis & Pancreatitis & & \\
Pleurisy & Peptic ulcer disease & & \\
Pneumonia & & & \\
Pneumothorax & & \\
Pulmonary embolism & & & \\
\hline
\end{tabular}


This represents the first published case of spasms of thoracic musc-lulature due to a hemorrhagic duodenal ulcer. In this case, the patients' thoracic spine spasms and scapular pain were erroneously attributed to discogenic disease. Schellhas et al (10) analyzed retrospectively the results of thoracic discograms performed on 100 outpatients. They found that the clinical presentation(s) of thoracic disc disease are highly variable and often mimic visceral conditions. In retrospect, there was no clue to demonstrate this patient had intra-abdominal pathology.

\section{CONCLUSION}

In conclusion, hemorrhagic ulcers should be included in the differential diagnosis when a patient presents with thoracic spasms without concurrent back pain. This represents the first published case of spasms of thoracic muscles due to a hemorrhagic duodenal ulcer. In this case, the patients' thoracic spine spasms and scapular pain were erroneously attributed to discogenic disease.

\section{REFERENCES}

1. Bonica JJ: The Management of Pain. Philadelphia, Lea \& Febiger, 1990.

2. Fischer AA: New approaches in treatment of myofascial pain. Phys Med Rehabil Clin North Am. 1997, 8:153-169.
Fields HL: Pain. New York, McGraw-Hill, 1987.

Holtmann G, Goebell H, Talley N: Functional dyspepsia and irritable bowel syndrome: is there a common pathophysiology basis. Am J of Gastroenterology. 1997, 6:954-959.

5. Reuben S, Steinberg R: Gastric perforation associated with the use of celecoxib. Anesthesiology. 1999, 91:5.

6. Sanson TG, O'Keefe KP: Evaluation of abdominal pain in the elderly. Emerg Med Clinics North Am. 1996, 3:615-627.

7. Zimmerman J, Siguencia J, Tsvang E: Upper gastrointestinal hemorrhage associated with cutaneous application of diclofenac gel. Am J of Gastoenterology. 1995, 11:2032-2034.

8. Guyton AC: The Textbook of Medical Physiology. Philadelphia, W. B. Sauders 1991, pp. 525-526.

9. Schneck, CD: Phone interview on anatomic pathway of referred visceral pain. July 20, 2000. Professor of Anatomy and Diagnostic Imaging. Temple University School of Medicine, Philadelphia, PA.

10. Schellhas KP, Pollei SR, Dorwart RH: Thoracic discography: A safe and reliable technique. Spine. 1994, 18:2103-2109. 